\title{
MEDIO AMBIENTE URBANO. CONCEPTUALIZACIÓN Y APLICACIÓN A LA CIUDAD DE PUERTOLLANO (CIUDAD REAL)
}

\author{
POR \\ MARÍA DEL CARMEN CAÑIZARES RUIZ
}

\begin{abstract}
Introducción: análisis geográfico, medio ambiente y espacio urbano
\end{abstract}

Resulta obvio que el análisis geográfico, preocupado por investigar las relaciones entre el hombre y el espacio, tiene importantes puntos de encuentro con el medio ambiente, entendido éste como simbiosis entre los componentes naturales y artificiales que dan soporte al hombre y a la propia sociedad humana (Valenzuela, M., 1984, 275). De hecho, para algunos autores, la propia geografía es, en esencia y por definición, «ambiental» (Hernández del Águila, R., 1997, 251), e incluso el término medio ambiente (environment/environnement), como afirma P. George, se emplea con significados muy parecidos al de medio geográfico (Clemente, E., 1972, 7).

Las relaciones entre ambos, tradicionalmente, han venido determinadas por el espacio natural (Unwin, 1992), pero no por ello es menos cierto que lo ambiental puede identificarse, también, con el espacio humanizado o artificial ya que el hombre es capaz de crear medios ambientes de naturaleza cultural o social y de modificar el medio natural

María del Carmen Cañizares Ruiz: Departamento de Geografía y Ordenación del Territorio. Universidad de Castilla-La Mancha.

Estudios Geográficos, LXII, 242, 2001

$$
-29-
$$


(Higueras, A., 1989, 155). En este sentido, las ciudades como construcciones artificiales del hombre poseen una dimensión ambiental.

Sin embargo, los lazos de unión geografía/medio ambiente no son nuevos ni actuales, más bien tienen una larga tradición. La propia evolución del hombre en el espacio los justifica. Como precedente encontramos la corriente ambientalista que tuvo vigencia desde mediados del siglo pasado hasta el último tercio de este siglo $^{1}$, y que ahora pretende retomarse en algunos sectores científicos. Autores destacados fueron E. Reclus, que incidiría en la influencia humana sobre la vegetación, los suelos y la dinámica fluvial o J. Brunhes que, a comienzos de siglo, introduciría en geografía humana el término «Raubwirtshaft» (economía depredadora) anunciado por el geógrafo Friedrich en 1904 y que tan variadas aplicaciones tiene en la actualidad. Con posterioridad, seguirán esta línea A. Voeiron, N. Sheler, C. Sauer y H. Barrows, quien define la geografía como ecología humana (Sauri, D., 1993, 148).

La institucionalización de la geografía como ciencia favoreció las interrelaciones, siendo objeto de diversos enfoques metodológicos. Eso sí, estuvo potenciada por la aparición de ciertos acontecimientos que habían transformado las relaciones entre el espacio y el hombre como, por ejemplo, la Revolución Industrial. Enriquecieron este panorama las numerosas reuniones y congresos que, bajo la óptica pluridisciplinar, se plantean un objetivo común: la protección y conservación de la naturaleza durante el siglo xx. En nuestro caso concreto la Unión Geográfica Internacional (U.G.I.) crea, con esos fines, la Comisión «Man and Environment» en 1968 (sustituida en 1976 por la Comisión de Problemas Ambientales) ${ }^{2}$.

No obstante, a partir de los años 60 esta corriente se sumió en una profunda crisis, cuando la precarización de la tradición ambientalista coincidió con la primera ola de los movimientos sociales preocupados

${ }^{1}$ G. Perkins Marsh escribe en 1864 una obra pionera en este sentido, Man and Nature. En ella se recogía la siguiente afirmación: «It is not the earth that made man but who made the earth» (no fue la Tierra quien creó al hombre sino el hombre quien creó la Tierra) destacando el papel activo del hombre como transformador del medio (Sauri, D., 1993, 144).

${ }^{2}$ Importantes son, también, las apariciones del Comité Consultivo sobre Recursos $\mathrm{Na}$ turales (1965) bajo los auspicios de la F.A.O. y de la O.M.S., y de diversas federaciones internacionales de ONG's como la Unión Internacional para la Conservación de la Naturaleza y de los Recursos Naturales (U.I.C.N.) en 1948, así como de organizaciones internacionales como el Fondo Mundial para la Naturaleza (W.W.F.) en 1961, Greenpace en 1971 o la asociación Amigos de la Tierra Internacional en 1971, también.

Estudios Geográficos, LXII, 242, 2001 
por el medio ambiente, sobre todo en los países anglosajones. Tal vez por ello, como afirman algunos autores, la geografía no debería dejar escapar la segunda oportunidad que plantea la crisis ecológica actual y sumarse decididamente al resto de las comunidades científicas interesadas por el medio ambiente (Sauri, D., 1993, 141).

Sin duda, la puesta en escena de diversos acontecimientos, con repercusiones ambientales ha reactivado el panorama investigador, incidiendo en una mayor preocupación por estos temas y, sobre todo, ha cuestionado el modelo de desarrollo actual, heredado del crecimiento conómico desenfrenado que surge a partir de la II Guerra Mundial ${ }^{3}$. Como es sabido, acontecimiento espacialmente relevante fue la celebración, en 1972, de la Conferencia de Estocolmo bajo el título «el hombre y su medio: bases para una vida mejor» (ONU), en la que se enuncian los principales factores de la problemática medioambiental. Seguidamente se inician los Programas de las Naciones Unidas para el Medio Ambiente (P.N.U.M.A.) y para el Desarrollo (P.N.U.D.) y aparece el Informe del Club de Roma,Los límites del crecimiento, advirtiendo del desorbitado aumento demográfico y su relación con los recursos (Barceló i Pons, B., 1984, 4 y ss.). En la década siguiente se reabre este debate y, en 1983, se crea la Comisión Mundial sobre Medio Ambiente y Desarrollo (W.C.E.D.) con el propósito de idear estrategias a largo plazo para conseguir modelos de desarrollo y elaborar recomendaciones para la mejora de la cooperación internacional. Sus trabajos se concretan en 1987 con la publicación del Informe Brundtland titulado Nuestro Futuro Común en el que aparece un concepto clave, «desarrollo sostenible», haciendo referencia al desarrollo que «satisface las necesidades de la generación presente sin comprometer la capacidad de las generaciones futuras para satisfacer sus propias necesidades» (Comisión Mundial del Medio Ambiente y Desarrollo, 1988, 10).

Actualmente, la llamada revolución verde, a la que a veces asistimos perplejos, ha contribuido a que las cuestiones medioambientales alcancen uno de los lugares privilegiados del panorama científico mundial, desgraciadamente, a consecuencia de la extrema necesidad de proteger el medio que nos rodea y de postular soluciones urgentes. A raíz de es-

${ }^{3}$ Destaca, especialmente, la declaración de 1970 como «Año de Protección de la Naturaleza», la fundación del Instituto Internacional para el Medio Ambiente y el Desarrollo (I.I.E.D.) y, sobre todo, el inicio del Programa «Men and the Biosphere» de la UNESCO, en 1971 . 
tos hechos, se suceden numerosas políticas medioambientales interesadas en crear un modelo de producción y consumo que sea asumible ecológicamente por el medio natural en el que vivimos y del que vivimos (Clemente, E., 1992, 10). En nuestro entorno inmediato, tal vez la más destacada sea el Programa Comunitario de Política y Actuación en materia de Medio Ambiente y Desarrollo Sostenible, vigente desde 1993 y que se apoya en una amplia normativa en favor de la protección y conservación.

Por último, si nos detenemos en el tercero de los elementos que aparecen en este epígrafe introductor, podemos definir el espacio urbano como una categoría que nace en la interfase de un modo específico de formación social y de un territorio, en un momento concreto que forma parte de un proceso histórico. Este espacio geográfico que calificamos como urbano está determinado por la existencia de la ciudad y, víctima de una creciente complejidad, hoy se ha convertido en objeto de investigación multidisciplinar.

Tras la expansión de los años 80, el «ciclo de recesión» (Chaline, C., 1995,35 ) o momento de crisis que viven hoy las ciudades, contribuye al replanteamiento de los modelos de desarrollo a fin de conseguir entornos más habitables y asegurar el bienestar de sus habitantes. Las cuestiones ambientales, dentro del marco del desarrollo sostenible, han alentado nuevas líneas de investigación geográfica que prestan especial atención a los muchos y graves problemas que padecen estos espacios.

El medio ambiente urbano como entorno material constituye uno de los factores más importantes en el proceso de formación del espacio urbano, no siempre considerado en su justa medida. Hoy se le presta una atención en otros momentos menospreciada por parte de la comunidad científica, debido al nivel de percepción desarrollado por la sociedad actual acerca de la calidad de vida de aquellos que habitan las ciudades.

Un concepto complejo. El medio ambiente urbano

Algunos términos básicos. Introducimos, desde el punto de vista geográfico, en un concepto tan versátil y complejo como el de medio ambiente urbano requiere tener presentes otros términos que, siendo afines, completan su panorama interpretativo. Se hace imprescindible carac-

Estudios Geográficos, LXII, 242, 2001

$$
-32-
$$


terizar conceptos que derivan tanto de la ecología ${ }^{4}$, como la ciencia estudia las relaciones de los seres vivos con el lugar que habitan, como del medio ambiente, es decir, del propio hábitat de los seres vivos. Teniendo en cuenta que el espacio urbano es nuestro objeto último de estudio intentaremos relacionar ambos términos con las implicaciones que pudieran tener en las ciudades.

En el ámbito de la ecología el concepto más cercano al de medio ambiente urbano es de ecología urbana, considerada para algunos como una ciencia social y para otros sólo como una técnica de investigación sociológica. Surge al aplicar la Teoría de la Ecología Humana ${ }^{5}$, preocupada por el modo en que las poblaciones humanas se organizan al objeto de mantenerse en su medio ambiente (Hawley, A. H., 1991, 25), a los espacios urbanos. Es decir, se centra, en las relaciones hombre/ciudad, las cuales dan lugar a fenómenos de competencia personal, dominación e invasión-sucesión. Su característica principal es la de considerar el espacio urbano como una comunidad de seres con una vinculación territorial, encontrando, en este aspecto, puntos de enlace con la geografía urbana. La ciudad, en sí misma, puede considerarse, entonces, como un fenómeno ecológico dada su naturaleza sociológica y cultural, así como la función integradora que desempeña por su condición de espacio para la convivencia integradora que desempeña por su condición de espacio para la convivencia (Higueras, A., 1989, 160).

El segundo de los términos, más amplio y hoy utilizado hasta el exceso, es el de medio ambiente. La Conferencia de Estocolmo (1972) lo definió como «el conjunto de elementos físicos, químicos, biológicos y de factores sociales capaces de causar efectos directos e indirectos, a corto o a largo plazo, sobre los seres vivientes y las actividades humanas» (Vázquez, L., 1991, 36). Actualmente lo identificamos con todo aquello que nos envuelve y es capaz de inferir en nuestra vida, a través de nuestras necesidades (materiales o intelectuales).

En él se integran tanto el medio natural, conformado por el suelo y el subsuelo, el aire, las aguas continentales superficiales y subterráneas, las marítimas, las costas, las playas y plataforma continental, la

\footnotetext{
${ }^{4}$ Su objeto de estudio está determinado por cómo los seres en crecimiento y multiplicación en un medio en constante cambio pero siempre restringido, cuya vida es una lucha continua de adaptación del individuo al medio (Hawley, A. H., 1974, 251).

${ }_{5}$ Nace en los años 20 abanderada por los sociólogos de la Escuela de Chicago, E. W. Burgucss y R. D. Mackenzic.
} 
flora, la fauna y la vegetación, los espacios naturales continentales submarinos y subterráneos y, en general, todos los elementos que forman parte de la biosfera; como el medio humano, construido por el entorno socio-cultural del hombre, y, en especial, los asentamientos humanos.

Esta diferenciación clara en dos ámbitos, el natural y el humano, nos permite hablar del medio ambiente humano y del medio ambiente natural. El primero plantea ciertas dificultades ya que se trata de un concepto, hasta ahora, poco utilizado. Principalmente, porque, en cuestiones ambientales, es muy difícil, y no siempre conveniente, separar la vertiente natural, relacionada con los caracteres físicos de un determinado espacio que condicionan y posibilitan la vida de las especies que lo ocupan, de la vertiente social, en la que sobresalen los aspectos humanos que configuran una determinada calidad de vida. Respecto al segundo es necesario decir muy poco puesto que su contenido se identifica con facilidad; incluso cuando nos referimos al medio ambiente, erróneamente, la mayor parte de las veces nos estamos refiriendo al medio ambiente natural.

El Medio Ambiente Urbano. Mucho más utilizado y, sin duda más relevante para el análisis que ahora plantearnos, es el término de medio ambiente urbano (M.A.U.), síntesis de los anteriores en su aplicación a un lugar concreto, la ciudad. Continúa siendo muy válida la definición del profesor Valenzuela como el «medio de vida cotidiana de los ciudadanos, síntesis entre el medio ecológico interactivo (sociedad-soporte) y la idea de vida cotidiana» (Valenzuela, M., 1984, 1997). Es decir, un sistema múltiple determinado por diferentes elementos: medio ecológico urbano (medio físico), medio construido (paisaje urbano, entorno edificado, ...) y medio social o socioeconómico (población, funciones económicas, diferenciación social, ...).

Las relaciones dentro de este «sistema autocontenido, altamente interrelacionado» (Perloff, H., 1973, 19) se caracterizan por el desarrollo de una dinámica que genera una compleja problemática: contaminación urbana (aire, agua, ruido, suelos, residuos), incremento más o menos desordenado o irregular del entorno edificado (calles, edificios, espacios abiertos, zonas de recreo) y competencia frente a la naturaleza (vegetación y vida silvestre en la ciudad). Y es ésta la razón por la que el acercamiento al medio ambiente en espacios urbanos frecuente-

Estudios Geográficos, LXII, 242, 2001

$$
-34-
$$


mente se realiza al analizar la problemática ambiental ${ }^{6}$, aunque este planteamiento no sea del todo correcto.

Actualmente, cuando más del $50 \%$ de la población mundial vive en ciudades, la búsqueda de lugares habitables que aseguren el bienestar de los ciudadanos se plantea con prioridad. Los denominados «problemas marrones» (básicamente, agua y saneamiento, contaminación atmosférica y residuos) parecen haberse intensificado en el momento actual. De ellos se derivan graves consecuencias y en definitiva, un medio dañado. A escala mundial, son dos los desafíos en relación con el medio ambiente urbano. El primero de ellos, tratar de mejorar las condiciones ambientales de los pobres que residen en las ciudades de los países en vías de desarrollo, y en segundo, lograr que las ciudades puedan compaginar las competitivas demandas del crecimiento económico con la protección ambiental (Muñoz, A., 1998, 2).

Es clara la ineficacia del modelo urbano actual, independientemente del nivel de desarrollo de cada territorio. La identificación de estos asentamientos con ecosistemas desorganizados y en desequilibrio con su medio ambiente (natural y social) incide en la búsqueda de ideas nuevas y soluciones a corto y medio plazo, en base a dos factores fundamentales: la presión que ejercen las actividades humanas en el medio urbano y el aumento de la movilidad. La rehabilitación ecológica de las ciudades exige hacer de ellas espacios útiles para satisfacer las verdaderas necesidades de sus habitantes, firmes en relación con su medio ambiente y bellos, no sólo desde el punto de vista de la estética oficial, sino por la adecuación al sitio en el que se localizada (Hernández, A., 1997, 19).

El medio ambiente urbano, como uno de los agentes creadores del paisaje urbano y también, como elemento de ordenación dentro de la ciudad, se encuentra en una situación delicada. Es un elemento en el que hay que detenerse especialmente porque es fundamental para asegurar la vida en óptimas condiciones a sus habitantes. Dentro del marco de crisis en el que se encuentran los espacios urbanos, «el M.A.U. nos permite ver el he-

\footnotetext{
${ }^{6}$ Pérdida de terreno por parte de los ecosistemas naturales y agrícolas en favor de las áreas urbanas, cambios en los sistemas atmosféricos regionales y globales, producción de enorme cantidad de desechos y vulnerabilidad de los espacios urbanos frente a las carencias de energía, agua, alimentos, aparición de enfermedades, etc., son algunas de las consecuencias ambientales del intenso crecimiento urbano en el Mundo (M.O.P.T., 1989, 37).
} 
cho urbano desde una perspectiva más amplia, como un sistema donde conceptos como energía, efecto invernadero, ruido, consumo energético, tienen un sitio junto a la transmisión cultural, la emigración, el mestizaje y el paro» (Alguacil, J.; Denche, C., y Hernández, A., 1994, 494).

Las aportaciones institucionales han sido fundamentales para contextualizar este elemento y prestarle la atención que merece. Seleccionando sólo las más relevantes comenzaremos citando el Manifiesto Europeo sobre el M.A.U. (1987) redactado por representantes de siete grandes ciudades europeas (Bonn, Copenhague, Londres, Lisboa, Roma, París y Madrid) (Ayuntamiento de Madrid, 1987, 8-10). Su principal aportación es la de constituir uno de los primeros avisos oficiales sobre la gran importancia del medio ambiente urbano y, sobre todo, llamar la atención para que los parámetros medioambientales sean contemplados en los procesos de planificación urbana. Destaca, especialmente, la identificación de la ciudad como ecosistema, como un sistema de relaciones entre elementos bióticos y abióticos aplicado a un espacio concreto. Ello nos permite hablar de un ecosistema urbano ${ }^{7}$ en el que existe una comunidad de organismos vivos (entre los que predomina el hombre), un medio físico que se transforma continuamente y un funcionamiento a base de intercambios de materia, energía e información (Rueda, S., 1993, 89), conformado por otros subsistemas secundarios (vegetal, animal, ...) y cuya complejidad es enorme.

Pero, sin duda, la aportación más destacable es el El Libro Verde sobre el Medio Ambiente Urbano (1990) que se inserta a modo de documento globalizador en las políticas sectoriales de la UE iniciadas en la década de los 90 . En él, partiendo de dos objetivos básicos, crear o recrear las ciudades para proporcionar entornos atractivos a sus habitantes y reducir su contribución a la contaminación global, aparecen enunciados dos principios: 1. aún estamos a tiempo de salvar la ciudad europea y 2. es necesario el compromiso tradicional de Europa en lo que ahora llama «cohesión social». (Comisión de las Comunidades Europeas, 1990, 48 y ss.).

La presión que ejercen muchas de las actividades que se concentran en la ciudad y la estructuración de nuestras zonas urbanas actúan como factores que inciden en los problemas del M.A.U., mientras que direc-

${ }^{7}$ En otras ocasiones aparece definido como ecosistema de aprovechamiento humano (Brookffield, H., 1982, 416).

Estudios Geográficos, LXII, 242, 2001

$$
-36-
$$


trices (coordinación integrada, responsabilidad, sostenibilidad y subsidiariedad), instrumentos (legislación, investigación, asistencia financiera, ...) y ámbitos de actuación (planificación urbana, transporte urbano, gestión de las aguas, industria urbana, ...) completan el marco de referencia para la planificación.

Este documento, aporta una concepción global del espacio urbano y ofrece una propuesta, en firme, de prestar atención a este elemento de vital importancia como es el medio ambiente en la ciudad, sin duda, olvidado en las gestiones y políticas urbanas y urbanísticas. Después de su aparición se han realizado diversos contactos tanto en el ámbito internacional como a escala europea, que pretenden seguir la filosofia expuesta, en especial, El Libro Blanco sobre el Medio Ambiente Urbano y el consecuente Plan de Acción para las Ciudades de Europa.

A partir de estas actuaciones, en su mayor parte instrumentos políticos que intentan paliar los problemas ambientales, se impone progresivamente el modelo de ciudad ambiental bajo los principios de sostenibilidad y de biodiversidad. La I Cumbre de la Tierra (Río, 1992) con el lema «Medio Ambiente y Desarrollo», consolida esta tendencia, planteando los principios de actuación de una forma drástica, entre los que destaca la protección del medio ambiente como una parte del proceso de desarrollo. Dentro de la Agenda 21, este foro presenta el Plan de Acción para el siglo XXI en el que se plantea el desarrollo como una búsqueda de la calidad de vida sin entrar en conflicto con el sistema que lo sostiene. Por su parte, II Cumbre de la Tierra (Nueva York, 1997) ha puesto en evidencia la lentitud con la que avanzan estos progresos, dada la falta de acuerdo y de compromiso de algunos de los países más poderosos del Mundo.

Dentro del marco europeo destacamos la Carta de Aalborg o Carta de las Ciudades Europeas hacia la Sostenibilidad (1994) con la que se inicia el Programa de Ciudades Europeas Sostenibles. En ella se establece un compromiso de actuación basado en tres acciones o partes: 1. una declaración de consenso de las ciudades europeas hacia el desarrollo sostenible; 2. el inicio de la Campaña de Ciudades Europeas Sostenibles y 3. la participación en las iniciativas locales del Programa 21 (Planes de Acción Social en favor de la sostenibilidad) ${ }^{8}$.

${ }^{8}$ En el ámbito internacional destacamos también el Programa de Ciudades Saludables que promueve la O.M.S. a partir de 1984, alentando la participación ciudadana en las cuestiones ambientales urbanas. Más adelante, en 1994, destaca la Carta de Megárides en la 
Más adelante, Estambul acoge en 1996 la Segunda Conferencia sobre Asentamientos Humanos (Hábitat II) en la que se proponen dos principios, vivienda adecuada para todos y consolidación de asentamientos urbanos sostenibles, configurando el Catálogo de Buenas Prácticas, donde destacan las áreas urbanas que llevan a cabo líneas de actuación en equilibrio con el medio ${ }^{9}$. También, ese mismo año, en Portugal, se celebra la Segunda Conferencia Europea sobre Ciudades Sostenibles, siendo diversas las reuniones e informes realizados en este sentido.

Para finalizar podemos decir que a lo largo de este último cuarto del siglo xx se ha avanzado en la concepción existente sobre el medio ambiente urbano. Como afirma el profesor Panadero se ha pasado desde la «ecologización de lo urbano» a la progresiva «ambientalización de lo urbano», llegando, finalmente, una «socialización de lo ambiental» donde priman conceptos como equidad, cohesión social o solidaridad (Panadero, M., 1998, 81).

\section{Ciudad y Medio Ambiente Urbano.}

El caso de Puertollano (Ciudad Real

Breve sintesis del desarrollo urbano y de su situación actual. La ciudad de Puertollano es, en la actualidad, el segundo núcleo urbano en importancia demográfica y socioeconómica de la provincia de Ciudad Real. Con 50.772 habitantes (Padrón Municipal de 1996) mantiene un lugar destacado como ciudad de primer orden dentro de la jerarquía urbana castellano-manchega (Cañizares, M. C., 1999b, 88). Su principal nota distintiva es la de constituir una de las pocas ciudades con tradición industrial dentro de un territorio altamente ruralizado, carácter atípico que le confiere un importante atractivo para la investigación geográfica. Su expansión y vertiginoso crecimiento desde la segunda mitad del siglo XIX hasta hoy está relacionada con la rentabilidad obtenida al explotar

que se presentan los principios fundamentales de la Ciudad de la Paz y de la Ciencia, de la Ciudad del siglo xxI en la que el medio ambiente en las ciudades deberá responder a las exigencias de los ciudadanos y del avance cultural y tecnológico.

9 En el caso español destacan las actuaciones llevadas a cabo en ciudades como Alcobendas, Girona, Alcudia, Barcelona, Calviá, Córdoba, San Sebastián o Palma de Mallorca (Ministerio de Fomento, 1996).

Estudios Geográficos, LXII, 242, 2001

$$
-38-
$$


y transformar las materias primas existentes en sus inmediaciones (aguas minero-medicinales, carbón y pizarras bituminosas) u otras traídas del exterior (petróleo y gas natural). Consecuentemente sus parámetros ambientales alcanzan un grado de complejidad inusual para otros núcleos de su entorno territorial.

Economía, población y espacio urbano son los tres elementos en base a los que podemos distinguir diversas etapas en el desarrollo urbano de este núcleo (Cañizares, M. C., 1998). Partiendo de unos orígenes inciertos podemos calificarlo como asentamiento estable durante el medievo cuando el sector de la meseta en el que se inserta fue repoblado por la Orden de Calatrava, después de la Reconquista (finales del siglo XII y comienzos del siglo XIII) ${ }^{10}$. La orientación socioeconómica de este núcleo rural era agropecuaria y estaba relacionada con la riqueza en pastos de la zona, área de tránsito para el ganado trashumante que bajaba desde el norte al sur de la Península. Artesanía y manufacturas (confección de paños) completaron, temporalmente, la base económica de una población que, con altibajos, no alcanzaba los 2.000 habitantes

Las transformaciones ocurridas a mediados del siglo XIX proporcionan un giro importante a este asentamiento. A partir de este momento podemos hablar de una ciudad minera que sustituye la ganadería y la agricultura como bases de la subsistencia de su población por la explotación de las aguas ferruginosas existentes en el subsuelo (lo que la convierte, momentáneamente, en una «ciudad balneario» ${ }^{11}$ ) y, a partir de 1873, por las explotaciones mineras de carbón de hulla. Esta segunda actividad, hoy enormemente arraigada, desencadena no sólo un cambio de función sino la diversificación socioeconómica de un núcleo que se separa claramente de los de su entorno, un crecimiento demográfico desorbitado que se materializa en sucesivas oleadas inmigratorias y, lo más importante, un desarrollo urbano espectacular.

La minería del carbón hizo crecer la economía local alcanzando su momento de mayor auge durante la I Guerra Mundial, cuando la imposibilidad de importar carbón inglés en Europa favorece a esta cuenca.

${ }^{10}$ El primer documento escrito en el que aparece citada Puertollano (el Puertoplano) se data en el año 1245 y corresponde a una Concordia firmada entre el Maestre de la Orden de Calatrava y el Arzobispo de Toledo.

${ }^{11} \mathrm{El}$ auge de Puertollano como «ciudad-balneario» se limita a la década de los 50 del siglo XIX, cuando recibía a numerosos enfermos que llegaban para «tomar las aguas». Entre ellos destacó un personaje relevante, el General Narváez, Presidente del Consejo de Ministros, cuyas visitas potenciaron diversas mejoras urbanísticas. 
Pero su población, 20.083 habitantes en 1920 (Censo Oficial de Población), sería testigo más adelante de un nuevo giro económico propiciado, en gran parte, por la explotación de las pizarras bituminosas interestratificadas entre las capas de carbón. En 1942 el Estado, mediante el recientemente creado Instituto Nacional de Industria, decide instalar en Puertollano una gran destilería de pizarras con el fin de obtener aceites industriales y combustibles, especialmente valiosos en período de autarquía ${ }^{12}$. Este hecho nos permite abandonar el calificativo de ciudad minera, aunque el carbón se siguió explotando, y utilizar el de ciudad industrial dando paso a una nueva fase caracterizada por acoger las grandes transformaciones responsables del desarrollo urbano actual.

Una segunda oleada inmigratoria que permite alcanzar los 50.003 habitantes en 1970 (Censo Oficial de Población) y un crecimiento determinado por la adaptación de las edificaciones a la topografia y la convivencia de urbanización de autoconstrucción con barriadas planificadas, son sus señas de identidad. La industria aparece ahora como principal protagonista, aunque experimenta cambios importantes durante la segunda mitad de los 60 y comienzos de los 70 cuando el agotamiento de las pizarras obligara a reorientar el Complejo Industrial hacia el refino de petróleo, la producción química y energética.

Llegada la crisis asistimos a la última etapa de evolución que calificamos como la ciudad postindustrial. En ella el crecimiento demográfico se estanca y la economía industrial sufre transformaciones internas muy relevantes. Destaca una creciente terciarización y un problema de paro más que acuciante, sólo paliado con algunas actuaciones como la llegada de la línea de alta velocidad ferroviaria en 1992 o la construcción de un segundo grupo térmico, ese mismo año. La expansión urbana se paraliza y el escaso crecimiento se limita a zonas septentrionales. Puertollano paga, sin duda, las consecuencias de un desarrollo urbano tan desorbitado. Consecuencias que la planificación municipal, tardía como en otros muchos casos, no puede paliar (el primer P.G.O.U. es de 1969).

Medio Ambiente Urbano en Puertollano. Caracterización y problemática. La situación ambiental que presenta actualmente la ciudad

${ }^{12}$ Las pizarras bituminosas las había comenzado a explotar, aunque a pequeña escala, la empresa francesa Sociedad Minero Metalúrgica de Peñarroya, una de las más importantes en la Cuenca de Puertollano.

Estudios Geográficos, LXII, 242, 2001

$$
-40-
$$


de Puertollano es resultado de su vertiginoso proceso de desarrollo. Si durante su primera fase de evolución podíamos encontrar un asentamiento en equilibrio con un medio muy poco alterado, será partir de mediados del siglo XIX cuando esta situación se complica. Como ejemplo durante la segunda fase, determinada principalmente por la explotación minera, este equilibrio empieza a resquebrajarse anunciándose importantes problemas relacionados con la infravivienda o la contaminación. Es, sin duda, el período que coincide con la ciudad industrial el que presenta una problemática más acusada, ya que las instalaciones industriales, aún exentas de controles normativos estrictos, son responsables de una contaminación urbana ciertamente relevante. Ese desequilibrio ambiental es heredado por la ciudad actual aunque minimizado por sistemas de control variados. Aun así la persistencia de una industria pesada altamente contaminante y de una concentración demográfica considerable son los responsables de la situación actual.

Para caracterizar el medio ambiente urbano en un espacio como éste, cuyos parámetros son semejantes al de otras muchas ciudades de tradición minero-industrial, partimos de identificarlo con el ámbito en el que se desenvuelve la vida cotidiana de los ciudadanos, síntesis entre el medio ecológico interactivo (el soporte físico y el modelo de organización social) y la calidad de vida urbana (Campesino, A. J., 1993, 251). Ello nos permite definir el perfil ambiental en base a tres elementos: el medio natural, el medio edificado y el medio social cuya síntesis aparece reflejada en el Cuadro I.

En esta caracterización que realizamos al determinar el perfil ambiental bajo el enfoque de la geografía urbana, se presenta al medio ambiente urbano como elemento conformador (determinante y determinado) de la ciudad y, por lo tanto, susceptible de ser analizado desde el punto de vista geográfico. Definir la problemática ambiental de este espacio y proponer posibles soluciones es el siguiente paso en el análisis del M.A.U. Somos conscientes de que contemplar estos problemas desde una perspectiva local y sectorial ofrece una visión muy limitada, por lo que es preciso ampliar la escala de análisis desde la ciudad al conjunto del planeta (Mulero, A., 1999, 25). Sin embargo es necesario también bajar a la realidad concreta de un espacio urbano y definir un posible patrón que pueda ser aplicado a otras ciudades. Este es el caso del estudio que sobre los problemas ambientales realizamos en Puertollano. 
CUADRo I

ELEMENTOS DE ANÁLISIS EN LA CARACTERIZACIÓN AMBIENTAL DE LA CIUDAD DE PUERTOLLANO

\section{Medio natural}

Características físicas del entorno territorial de Puertollano.

Análisis del clima local. Detección de la «Isla de Calor».

Las áreas verdes y la vegetación en la ciudad. La fauna urbana.

El agua y su tratamiento (abastecimiento, consumo, tratamiento, ...).

Los residuos y su tratamiento (tipos de residuos, producción, selección, ...).

$\mathrm{El}$ aire. La red de vigilancia de la calidad del aire en Puertollano.

\section{Medio edificado}

Evolución del espacio edificado.

Paisaje urbano y usos de suelo.

Infraestructuras y equipamientos.

La densidad urbana.

El patrimonio cultural urbano (minero-industrial).

La percepción del medio edificado.

\section{Medio social}

La evolución demográfica y social.

Zonificación socio-espacial. Grandes áreas urbanas.

La calidad de vida y su percepción (salud, deporte, alimentación, ocio, ...).

El proceso evolutivo experimentado por esta ciudad es el responsable del deterioro en la calidad del medio al que han de enfrentarse sus habitantes. De hecho, las ciudades «pueden considerarse, al mismo tiempo sujetos agentes y pacientes de los problemas ambientales» (Valenzuela, M., 1997, 21). En este caso, es evidente la existencia de un medio ambiente urbano degradado que presenta un claro déficit directamente relacionado con la concentración demográfica (tráfico, calefacciones, necesidad de recursos, ...) y, muy especialmente, debido a la ubicación en las proximidades del núcleo urbano de industrias altamente contaminantes (químicas, refino de petróleo, extracciones de carbón a cielo abierto, centrales termoeléctricas, ...).

$$
-42-
$$


La falta de datos comparables y la ausencia de un sistema de indicadores ambientales ${ }^{13}$ comúnmente reconocido y aplicable tanto a escala nacional como europea nos obliga a utilizar otros criterios para poder definir cuáles son los problemas más acuciantes. En este caso y teniendo en cuenta que analizamos un núcleo incluido en la Red Española de Ciudades Saludables ${ }^{14}$ hemos optado por aplicar los criterios requeridos por este Programa para que una ciudad se pueda considerar saludable $y$ en base a ello determinar los principales problemas ambientales de Puertollano (Cañizares, M. C., 1998, 560 y ss.).

Estos criteros, organizados en grupos (agua, contaminación atmosférica, residuos sólidos, alimentación, situaciones de emergencia, habitabilidad, promoción de salud y medio ambiente social), abarcan tres niveles: nivel $3 .^{\circ}$ o básico, en el que se incluyen indicadores imprescindibles para aplicar a una ciudad el calificativo de «saludable», tales como la dotación suficiente de agua potable a toda la población o la existencia de planes de recogida de residuos sólidos urbanos; nivel $2 .^{\circ}$ o recomendable, en el que se encuentran áreas que cumplen los de nivel 1 y otros específicos, tales como el control sanitario de las redes de distribución de aguas o el fomento de la clasificación y selección de residuos sólidos en origen; y nivel $1 .^{\circ} \mathrm{o}$ ideal que supone la consecución de los criterios anteriores, ampliándose a otros que permiten aplicar el calificativo de saludable (R.E.C.S., 1994), aquí encontramos, por ejemplo, el control de las radiaciones ionizantes o la elaboración de un mapa de riesgos. La extensión y contenido de los criterios contenidos en cada uno de estos niveles nos hace imposible reproducirlos en su totalidad.

Aplicados a la ciudad de Puertollano llegamos a una primera conclusión significativa y es que se encuentra «lejos del apelativo "saludable” (Cañizares, M. C., 1998, 584). Las razones se centran, básicamen-

${ }_{13}$ Existe, no obstante, una propuesta de indicadores para España en un intento de definir el conjunto de problemas ambientales mediante variables cuyo objetivo sea promover una visión totalizadora de los intereses predominantes relativos al medio ambiente (M.M.A., 2000, 19).

${ }_{14}$ El Programa «Ciudades Saludables» se inicia con la Carta de Otawa en 1984 promovida por los países miembros de la O.M.S. y bajo la necesidad de reorientar los servicios sanitarios y sus recursos hacia la promoción de la salud. De la red mundial se derivan otras supraestatales como la europea (1987), nacionales como la española (1988) y regionales como, en este caso, la castellano-manchega (1990). 
te, en el incumplimiento o el cumplimiento parcial de algunos de los criterios denominados de nivel $3 .^{\circ} \mathrm{o}$ básicos. Nos referimos a criterios relacionados, principalmente, con los grupos de contaminación atmosférica, residuos sólidos, alimentación, promoción de salud y medio ambiente social.

La aparición de frecuentes episodios de atmósfera contaminada, producidos esencialmente por concentraciones horarias de ozono, así como la imposibilidad de detectar las fuentes de emisión de estos contaminantes y proceder a la corrección de estas situaciones (aunque se presuponen pero no es posible asegurarlo) y, por último, la inexistencia de control alguno sobre los ruidos, excepto denuncias, permite destacar la contaminación atmosférica como uno de los principales problemas ambientales de Puertollano.

Siguiendo con el análisis de indicadores básicos, la frecuente aparición de vertederos incontrolados (a pesar de que se ha procedido a la clausura de muchos otros), la existencia de ventas clandestinas de productos alimenticios sin control, la aparición de focos de contaminación por animales no domésticos que cohabitan en casas (también clausurados cuando se detectan), la ausencia de un Plan de Emergencia Exterior en el complejo industrial o las carencias en la protección a la infancia y en la acogida de población en albergues, indicadores que se cumplen de forma insuficiente, son algunos ejemplos claros de la situación de déficit ambiental. Lamentablemente, si se incumplen algunos indicadores «básicos», también ocurre lo mismo con algunos recomendables como la adopción de medidas para limitar las emisiones contaminantes de fuentes difusas, $\mathrm{y}$, por supuesto, con algunos otros ideales corno la ausencia de un mapa de riesgos, sólo limitado a algunos supuestos.

Consecuentemente la problemática ambiental de la ciudad de Puertollano se puede agrupar de la siguiente forma:

\section{Contaminación urbana}

- Atmosférica, principalmente, de origen industrial.

- Acústica, relacionada con el modo de vida urbano (tráfico).

- Aguas, en relación a las actividades industriales.

- Olores, debido a la industria (petroquímica) y al depósito de residuos.

Estudios Geográficos, LXII, 242, 2001

$$
-44-
$$


Medio natural

- Necesidad de ampliación de las zonas verdes urbanas.

- Degradación del paisaje natural en el entorno afectado por las explotaciones mineras y por la industria.

- Riesgos derivados de la fauna. Especialmente en la convivencia con animales no domésticos.

Medio edificado

- Degradación del centro histórico, sometido a una drástica destrucción.

- Problemas de vivienda. Infravivienda en alguno barrios, costo, etc.

- Transporte (mercancias peligrosas) y tráfico urbano.

Medio social

- Desempleo, sobre todo, de población vinculada al sector secundario.

- Marginación y malos tratos.

- Problemas de salud (índices elevados de determinadas enfermedades).

La aplicación de soluciones a estos problemas implica una reconversión ecológica de esta ciudad que aún está por llegar y que debe abordarse no sólo desde el ámbito preventivo (control de contaminantes, mejora del mercado laboral, ...) sino también desde el correctivo (restauración del paisaje, limitación de emisiones, ...). En este sentido es destacable la actuación municipal a partir del período democrático, plasmada no sólo en un mejor entendimiento con las multinacionales que dominan el complejo industrial (lo cual podrá incidir en el control de la contaminación) sino en actuaciones concretas como la inclusión de esta ciudad dentro del Programa de Ciudades Saludables (1991) adscrita a la red regional de Castilla-La Mancha. A ello se suma la creación de una Red de Vigilancia de la Calidad del Aire (1991), que permite la recogida de datos en tiempo real, la aprobación de unas Ordenanzas Municipales de Protección Ambiental (1996) y la creación de un Consejo Local de Medio Ambiente (1996). En el ámbito empresarial, destacan las labores de restauración paisaje llevadas a cabo por la empresa minera Encasur, S. A. 
CuADro II

ÁMBITOS DE ACTUACIÓN DE LA PLANIFICACIÓN INTEGRAL DE MEJORA AMBIENTAL URBANA EN PUERTOLLANO

\section{Contaminación urbana}

Mejora en la calidad del aire. Control y reducción de la contaminación atmosférica en colaboración RVCAP y empresas contaminantes.

Control y gestión de los residuos y de las aguas.

Control de ruidos (incluyendo control de tráfico en las áreas centrales).

\section{Medio natural}

Protección y revalorización del entorno natural y de las áreas verdes (sobre todo zonas degradadas por la minería a cielo abierto).

\section{Medio edificado}

Protección y revalorización del patrimonio cultural, especialmente el de carácter minero-industrial cuyo potencial es elevado.

Fomento de una mayor divesidad urbana evitando la difusión espacial.

Revitalización de las zonas urbanas marginales o con problemas.

Gestión y aprovechamiento de la energía.

Atención a la industria (localización, contaminación...).

\section{Medio social}

Fomento de la participación ciudadana mediante campañas informativas. Iniciativas sociales de integración en colectivos marginales (gitanos, ...). Fomento del empleo, especialmente de carácter industrial y servicios. Cooperación interurbana.

Atención especial a la salud elaborando estudios que permitan relacionar contaminación atmosférica y determinadas enfermedades.

Estas «terapias» ambientales sólo pueden venir definidas por la planificación integrada, puesto que los actuales planes generales de urbanismo han resuelto muy poco al respecto. La mera división y clasificación de usos de suelo es insuficiente (Troitiño, M. A., 2000). La propuesta que, en su momento realizamos (Cañizares, M. C., 1998, 714 y ss.) y ahora ratificamos se enmarca en la filosofía de mejora ambiental

Estudios Geográficos, LXII, 242, 2001

$$
-46-
$$


propuesta por El Libro Verde del Medio Ambiente Urbano (1990) cuyos objetivos aplicados a este caso concreto deberán orientarse a maximizar la capacidad del medio urbano y minimizar los costes derivados de las actividades sociales y económico-productivas que causan impactos, previa elaboración lógica de un diagnóstico ambiental. Los factores de actuación vendrán dados por la consecución de un objetivo concreto, el de la mejora ambiental urbana, por una base local que plantea el propio término municipal, por la modificación del entorno en aquellas zonas que se requiera, por la sostenibilidad tanto ambiental como económica y, finalmente, por la condición de ser socialmente útil. Los instrumentos de gestión se relacionarán directamente con las líneas europeas de financiación y los ámbitos de actuación los detallamos en el Cuadro II.

\section{Conclusiones}

Como en el caso de otras muchas ciudades españolas, en Puertollano, los postulados de la civilización técnico-industrial y las tendencias de localización industrial, una práctica urbanística errónea, una vertiginosa expansión del transporte motorizado, las limitaciones de las tramas urbanas históricas, una actuación político-ambiental escasamente preventiva, rígida y muy sectorial, la tendencia a disfrazar los impactos ambientales trasladándolos fuera del perímetro urbano y un deficiente conocimiento del habitante urbano respecto a su entorno y una actitud hipócrita bastante extendida a la hora de contribuir a la mejora de éste (Mulero, A., 1999, 19) son las causas generales que encontramos a la hora de abordar la problemática ambiental. Si concretamos encontramos un desarrollo urbano intenso relacionado con las actividades minero-industriales cuyo potencial contaminante es elevado, una concentración demográfica que demandaba espacio construido cuando aún la planificación municipal no había llegado y una pasividad casi absoluta por parte de los ciudadanos y de las instituciones hasta fechas muy recientes. Por tanto, convertir esta ciudad, como muchas otras, en un espacio «habitable» 0 «sostenible», entendiendo por tal no sólo aquel que satisface sus necesidades actuales sin poner en peligro las futuras sino aquel que implica la mejora en la calidad de vida (Comisión Europea, 1996, 16-17) es el gran reto para el futuro. 
Se trata de una tarea atractiva a la vez que compleja en la que, no obstante los esfuerzos realizados en los últimos años son importantes. La planificación integrada es el instrumento más adecuado aunque, consideramos, también y en la línea del profesor Troitiño, que la eficiencia de esta planificación para alcanzar el desarrollo sostenible deberá implicar una territorialización de las decisiones económicas, aspecto para el que es absolutamente imprescindible fomentar la cultura territorial en favor de una mejora en las tomas de decisión y de la calidad de vida de los ciudadanos (Troitiño, M. A., 2000, 629-630).

Para finalizar cabe tan solo añadir que el Medio Ambiente Urbano debe ser entendido, adoptando una visión globalizadora, como un elemento/factor más del espacio urbano y de su proceso de desarrollo, con el cual está íntimamente relacionado (como hemos observado en el caso analizado). No obstante, nos asalta la duda de si, como afirma J. Bosque Maurel, la ciudad es habitable por definición ya que su objetivo básico es la supervivencia del hombre y plantearse esto puede parecer superfluo o peyorativo (Bosque, J., 1995, 7 y ss.). Coincidimos en que, tal vez los problemas haya que buscarlos en su propio desarrollo, en el proceso de urbanización sufrido, en la concentración demográfica, en el avance de los medios de transporte, etc. Quizás la clave está en que, la nueva urbe, como afirma este geógrafo, no es sino un complejo espacio ciudadano, diferenciado y especializado funcional y socialmente $\mathrm{y}$, en cierta forma, producido y sometido a una nueva forma de cultura regida por las novísimas tecnologías-punta, en concreto las relacionadas con la automoción, los flujos inmateriales y el proceso informático. Y a ello debe, en definitiva, el deterioro de su medio ambiente físico y social, en el que aparecen sobrevalorados conceptos como lo económico, el individualismo, o la insolidaridad.

Este análisis se presenta, por tanto, repleto de dificultades, aunque, a la vez, de interés. Es un campo de investigación en el que aún existe mucho trabajo por delante y si la tradición geográfica ha supuesto una aportación más en la línea de búsqueda de ideas, conceptos, métodos o modelos útiles para la consecución de una toma de conciencia crítica y compleja en relación a la actual crisis ecológica (Hernández del Aguila, R., 1997, 216) no podemos esperar menos de la geografía actual cuya predisposición hacia estas temáticas es indudable.

Estudios Geográficos, LXII, 242, 2001

$$
-48-
$$




\section{BIBLIOGRAFÍA}

AyUntamiento De MAdRID (1988): Medio Ambiente Urbano. Entorno Humano, Madrid. Exposición Casa de Vacas del Retiro (15-30 septiembre).

AlguACIL, J.; Denche, C., y HeRnÁNDEZ, A. (1994): «La eclosión de la sociedad Urbana: desorden o medio ambiente urbano", Rev. Ciudad y Territorio. EE.TT. N. ${ }^{\text {os }} 100-101$, Madrid, MOPTMA, pp. 487-498.

BARBIERI, G.; CANIGIANI, F., y CASSI, L. (1997): Geografia e Ambiente. Il mondo attuale e $i$ suo problemi, Torino, UTET Libreria.

BARCELO I PONS, B. (1984): «Fundamentos conceptuales de la preocupación geográfica por el medio ambiente», en VALENZUELA, M. (coord.): Geografía y Medio Ambiente, Madrid, MOPU, pp. 3-19.

Bosque Maurel, J. (1995): «La Ciudad "Habitable". Utopía o Realidad», Rev. Cuadernos Geográficos, Granada, Universidad de Granada, pp. 7-16.

BRookfIELD, H. (1982): «El Hombre y los Ecosistemas», Revista Internacional de Ciencias Sociales, n. ${ }^{\circ}$ 93, vol. XXXIV, n. ${ }^{\circ} 33$, París, UNESCO, pp. 415-435.

CAmpesino Fernández, A. J. (1993): «La política comunitaria sobre el medio ambiente urbano y su aplicación a Extremadura», en VV. AA.: Desarrollo Regional de Extremadura, Cáceres, Cámara de Comercio e Industria de Cáceres, pp. 251265.

CAÑIZAREs RuIZ. M. C. (1998): Desarrollo urbano y problemática ambiental de la ciudad de Puertollano (Ciudad Real), Cuenca, Servicio de Publicaciones de la Universidad de Castilla-La Mancha.

__ (1999a): «Puertollano: de la "ciudad del carbón" a la "ciudad del petróleo"», en DomíngueZ, R. (coord.): La ciudad. Tamaño y crecimiento, Málaga, AGE y Universidad de Málaga, pp. 75-84.

- (1999b): «La red urbana», en TAMAMEs, R., y HERAS, R. (coords.): Enciclopedia de Castilla-La Mancha, vol. II, El espacio humano, Madrid, Ed. Edicsa, pp. 77-95.

Clemente, E. (1992): «Geografía y medio ambiente», Rev. Situación 1991/2, Bilbao, Banco Bilbao-Vizcaya, pp. 7-15.

Comisión DE LAS COMUNIDADEs EuRopeas (1990): El Libro Verde sobre el Medio Ambiente Urbano, Bruselas, Luxemburgo.

- (1996): Ciudades Europeas Sostenibles. Informe, Bruselas, marzo de 1996, Grupo de Expertos sobre el Medio Ambiente Urbano.

Comisión Mundial del Medio Ambiente y del Desarrollo (1988): Nuestro futuro común, Madrid, Alianza Editorial.

Chaline, C. (1995): «Reflexión sobre la dinámica urbana del siglo XX», en GARCía BALLESTERos, a. (coord.): Geografía Urbana-1. La Ciudad: Objeto de Estudio Pluridisciplinar, Barcelona, Ed. Oikos-Tau, pp. 35-38.

FERRER REgales, M. (1981): «Introducción al estudio ecológico de las ciudades españolas», en VV. AA.: Estudios de Geografía Urbana, Pamplona, Homenaje a Alfredo Floristán, pp. $170-179$.

Hawley, A. H. (1974): «Ecología y Ecología Humana», en Theodorson, G. A. (ed.): Estudios de Ecología Humana, Barcelona, Ed. Labor, pp. 243-254.

- (1991): Teoría de la Ecología Humana, Madrid, Ed. Tecnos.

HERNÁNDEZ, A. (dir.) (1997): La ciudad de los ciudadanos, Madrid, Ministerio de Fomento, Dirección General de la Vivienda, la Arquitectura y el Urbanismo.

HERNÁNDEZ DEL ÁGUILA, R. (1997): «Perspectiva geográfica», en Novo, M., y LARA, R.: El análisis interdisciplinar de la problemática ambiental, vol. II, Madrid, UNED, Cátedra y UNESCO, pp. 213-257.

Higueras, A. (1989): «La Ciudad como fenómeno ecológico», Rev. Geographicalia, n. ${ }^{\circ} 26$ (diciembre), Zaragoza, Universidad de Zaragoza, pp. 155-163. 
Ministerio de Fomento (1996): primer catálogo español de buenas prácticas. Ciudades para un futuro más sostenible (Hábitat II), 3 vols. (2. ${ }^{a}$ ed.), Madrid.

MINISTERIO DE MEDIO AMBIENTE (M.M.A.) (2000): Indicadores ambientales. Una propuesta para España, Madrid, Dirección General de Calidad y Evaluación Ambiental.

M.O.P.T. (1989): El Hombre pertenece a la Tierra, Madrid, UNESCO, MAB.

Mulero, P. (1999): Introducción al Medio Ambiente en España, Barcelona, Ariel Geografía.

MuÑoz, A. (ed.) (1998): Recursos mundiales. La guía global del medio ambiente urbano, Madrid, Ministerio de Medio Ambiente. Instituto de Recursos Mundiales.

Panadero Moya, M. (1998): «Las ciudades y el medio ambiente», Rev. Anales del Centro de Albacete de la UNED, n. ${ }^{\circ} 14$, Albacete, pp. 67-82.

PARRA, F. (1994): «La Ciudad como Ecosistema», Rev. Ciudad y Territorio. EE.TT., n. ${ }^{\text {os }}$ 100-101, Madrid, MOPTMA, pp. 411-419.

PERLoff, H. (1973): La calidad del medio ambiente urbano, Barcelona, Ed. Oikos-Tau.

Pillet,F. (1995): «El medio ambiente urbano de Ciudad Real», Rev. Anales de Geografía de la Universidad Complutense, n. $^{\circ}$ 15; Ciudad y Medio Ambiente. Homenaje al profesor D. Joaquín Bosque Maurel, Madrid, Universidad Complutense de Madrid, pp. 527-537.

Red Española de Ciudades Saludables (R.E.C.S.) (1994): Criterios para Ciudades Saludables, Madrid, Federación Española de Municipios y Provincias.

Rueda, S. (1993): «La ciudad como ecosistema urbano. Criterios de ecología urbana para la planificación», rev. Espacio y Sociedad, n. ${ }^{\circ} 8$ (junio), Madrid, pp. 89-100.

SAURI, D. (1993): «Tradición y renovación en la Geografía Humana Ambientalista», Rev. Documents d'Analisi Geografica, n. ${ }^{\circ} 22$, Barcelona, pp. 139-157.

TRoitiño, M. A. (2000): «Ordenación del territorio y desarrollo territorial: la construcción de las geografías del futuro», en VV.AA.: Lecturas geográficas. Homenaje al profesor José Estébanez Álvarez, Madrid, Universidad Complutense de Madrid, pp. 629-642.

Unwin, T. (1992): El lugar de la Geografía, Madrid, Ed. Cátedra.

VALENZUELA, M. (1984): «El medio ambiente urbano: su conceptualización y problemática desde la óptica geográfica», en Valenzuela, M. (coord.): Geografía y Medio Ambiente, Madrid, MOPU, pp. 275-307.

- (1997): «Perspectiva Urbanística», en Novo, M., y LARA, R.: El análisis interdisciplinar de la problemática ambiental, vol. II, Madrid, UNED, Cátedra y UNESCO, pp. 19-106.

VÁzquez GuILlén, L. (coord.) (1990): Tratado sobre Medio Ambiente, 2 vols., Madrid, Ed. Lafer.

VV.AA. (1995): Carta de las Ciudades Europeas hacia la Sostenibilidad. La Carta de Aalborg, Bruselas, Campaña de Ciudades Europeas Sostenibles.

(1999): «Informe Especial. Ciudades y Medio Ambiente», Rev. Ecosistemas, vol. VIII, Madrid, pp. 35-56.

RESUMEN: Este artículo pretende aportar una aproximación al concepto de medio ambiente urbano así como una reflexión sobre su propio contenido como elemento y factor del desarrollo urbano. Su análisis se concreta en la ciudad de Puertollano en la que la concentración demográfica y la aparición de industrias altamente contaminantes (químicas, refino de petróleo, termoeléctricas) son las causas fundamentales de una extensa problemática frente a la cual sólo es válida una planificación integrada basada en la mejora ambiental urbana.

Palabras Clave: Medio ambiente urbano, Sostenibilidad urbana, Problemática ambiental.

Estudios Geográficos, LXII, 242, 2001

$$
-50-
$$


ABSTRACT: This paper tries to be not only an approach to urban environment conception, but also a refletion on its own content like element and factor of urban development. Its research centres on the city of Puertollano, where the demographic concentration and the appearance of highly polluant industries (chemistry, refining petroleum, thermoelectric) are the main reasons of a vaste set of problems. In order to the face them, the only viable solution is an integrated planning based on the environmental improvements.

KEY WORDS: Urban environment, Urban sustainability, Environmental problems.

REsumÉ: Cet article, il prétent apporter une approximation a l'idée ou concept du environement urbain, aussi bien q'une réflexion sur leur contenu comme un élement et aussi facteur du developpement urbain. Son analyse se precise a la ville de Puertollano dans laquelle la concentration demographique et l'apparition des industries hautement contaminants (chimiques, raffine du pétrole, thermoélectriques) ce sont les causes fondamentales d'une vaste problèmatique en face laquelle seulement est valide une planification intégrale base sur l'amélioration du milieu.

MoTS CLÉ: Environement urbain, Soutenabilité urbaine, Problèmatique du milieu. 